\title{
Clinical Events End Timepoint
}

National Cancer Institute

\section{Source}

National Cancer Institute. Clinical Events End Timepoint. NCI Thesaurus. Code C87849.

A point in time that indicates the conclusion of a clinical event. 